\title{
A Study Regarding Solution of a Knowledge Model Based on the Containing-Type Error Matrix Equation
}

\author{
Xilin Min, Kaizhong Guo, Jize Huang \\ School of Management, Guangdong University of Technology, Guangzhou, China \\ Email: chinamxl@126.com
}

Received May 19, 2012; revised June 22, 2012; accepted July 10, 2012

\begin{abstract}
An error matrix equation based on error matrix theory was presented in previous research of the error-eliminating theory. The purpose of solving the error matrix equation is to create a decision support on how to switch from bad to good status. A matrix based on error logic is used to express current status $u$, expectant status $u_{1}$ and transformation matrix $T$. It is $u, u_{1}$, and $T$ that are used to build error matrix Equation $T(u)=u_{1}$. This allows us to find a method whereby bad status " $u$ " changes to good status " $u_{1}$ " by solving the equation. The conversion method that transform from current to expectant status can be obtained from the transformation matrix $T$. On this basis, this paper proposes a new kind of error matrix equation named "containing-type error matrix equation". This equation is more suitable for analyzing the realistic question. The method of solving, existence and form of solution for this type of equation have been presented in this paper. This research provides a potential useful new technique for decision analysis.
\end{abstract}

Keywords: Error Matrix; Set Equation; Containing-Type Error Matrix Equation; Knowledge Model

\section{Introduction}

The truth is always expected; however, error should be avoided and eliminated firstly in order to seek truth. On the one hand, it should be known why and how the error arises with the aim of avoiding and eliminating errors; on the other hand, it should be known how to eradicate it when error appears. Moreover, within a faultless system, proposition and decision can also be naturally transferred into being defective in normal conditions, because of the passage of time, changes in natural conditions, or development of science. So, errors are required to be eliminated continuously.

Chinese scholar Professor Guo Kaizhong has found the error-eliminating theory since the 1980 s, and has taken the study of error to a new level.

The error-eliminating theory is an emerging subject, which is used to study error elimination [1]. The theory of error-eliminating defines the objective system, in which one error factor at least is included to characterize and eliminate errors. Error setting could be termed as $C=\{(u, x) \mid u \in U, X=f(G \neq>u, f \subseteq U \times R)\}$. As mentioned in the book "Theory of Error Sets" [2], all unary or multiple error sets have been defined in error matrix. In the book "Error Logic" [3], every element $(u, x)$ is expressed as

$$
\begin{aligned}
& \left(U, S(t), \boldsymbol{p}\left(\psi_{1}, \psi_{2}, \cdots, \psi_{n}\right), T(t), L(t),\right. \\
& \left.\left(x(t)=f\left((u(t), \boldsymbol{p}), G_{U}(t)\right)\right), G_{U}(t)\right)
\end{aligned}
$$

from the standpoint of logic theory, where $U$ is discussing domain; $S(t)$ is the objects of $u ; p\left(\psi_{1}, \psi_{2}, \cdots, \psi_{n}\right)$ is the space under consideration, and $\psi_{n}$ is used to define the dimensions in the space; $T(t)$ is the characteristic of the described objects; $L(t)$ is the value of $T(t) ; x(t)=f\left((u(t), \boldsymbol{p}), G_{U}(t)\right)$ is error function; $G_{U}(t)$ are decision rules. As shown in the above function, object $u(x)$ and rule $G_{U}(t)$ are set as independent variables. In addition to this, Guo Kaizhong, Liu Shiyong and Li Min [5-7] have studied Logical relationship among various types of error logical words.

Error matrix based on error logic is a useful method of modeling for scenes. $X, A$ and $B$ could be structured as an error matrix equation for three error matrix, such as $X A=$ $B$. $X A=B$ is just a concrete form of error matrix equation $T(u)=u_{1}$. By means of solving the error matrix equation, matrix $X$ of error logic transformation can be obtained, which can be used in reasoning regarding the question. $X$ allows us to find a method, in which bad status " $A$ " changes into good status " $B$ ". Guo Kaizhong and Min 
Xilin [8-10] have studied the solution of equation $X A=B$, in which a containing-type error matrix, such as $X A \supseteq B$, was found to be more suited for analyzing the actual question. In this paper, it is attempted to solve the containing-type error matrix in order to seek new ways of error-elimination.

\section{Error Matrix Equation}

\subsection{Equational-Type Error Matrix Equation}

In the previous research, all of the error matrix equations have been classified into two types (see Table 1). There are five kinds of equations in each type. Meanwhile, there are three constraints on the solution of the equation: The first is an objective condition, named " $k g$ "; the second is a stipulation that has been confirmed, named " $r w$ "; the third is the restriction of requirement, named " $x q$ ".
In common situations, these three restrictions above can be shown by certain sets. The factors in the matrix and the solving of the matrix equations are also measured in a certain set. Therefore, we can use the intersection method of solving sets which includes the matrix equation and ignores these three restrictions in solving the process.

\subsection{Containing-Type Error Matrix Equation}

In order to analyze practical issues, we propose a containing-type error matrix equation (see Table 2).

\section{Solving a Containing-Type Error Matrix Equation}

In the following, let us now consider how to solve the second type of Equation (1): $X A \supseteq B$.

$$
\begin{aligned}
& X=\left(u_{1}, x_{1}\right)=\left(U_{1}, S_{1}(t), \boldsymbol{p}_{1}, T_{1}(t), L_{1}(t), x_{1}(t)=f_{1}\left(\left(u_{1}(t), \boldsymbol{p}\right), G_{U 1}(t)\right), G_{U 1}(t)\right) \\
& =\left[\begin{array}{ccccccc}
U_{10 X} & S_{10 X}(t) & \boldsymbol{p}_{10 X}\left(\psi_{1}, \psi_{2}, \cdots, \psi_{n}\right) & T_{10 X}(t) & L_{10 X}(t) & x_{10 X}(t)=f_{10 X}\left(\left(u(t), \boldsymbol{p}_{10 X}\right), G_{U 10 X}(t)\right) & G_{U 10 X}(t) \\
U_{11 X} & S_{11 X}(t) & \boldsymbol{p}_{11 X}\left(\psi_{1}, \psi_{2}, \cdots, \psi_{n}\right) & T_{11 X}(t) & L_{11 X}(t) & x_{11 X}(t)=f_{11 X}\left(\left(u(t), \boldsymbol{p}_{11 X}\right), G_{U 11 X}(t)\right) & G_{U 11 X}(t) \\
& & \vdots & & & \\
U_{1 t X} & S_{1 t X}(t) & \boldsymbol{p}_{1 t X}\left(\psi_{1}, \psi_{2}, \cdots, \psi_{n}\right) & T_{1 t X}(t) & L_{1 t X}(t) & x_{1 t X}(t)=f_{1 t X}\left(\left(u(t), \boldsymbol{p}_{1 t X}\right), G_{U 1 t X}(t)\right) & G_{U 1 t X}(t)
\end{array}\right] \\
& A=\left(U_{2}, S_{2}(t), \boldsymbol{p}_{2}, T_{2}(t), L_{2}(t), x_{2}(t)=f_{2}\left(\left(u_{2}(t), \boldsymbol{p}_{2}\right), G_{\mathrm{U} 2}(t)\right), G_{\mathrm{U} 2}(t)\right) \\
& =\left[\begin{array}{ccccccc}
U_{20} & S_{20}(t) & \boldsymbol{p}_{20}\left(\psi_{1}, \psi_{2}, \cdots, \psi_{n}\right) & T_{20}(t) & L_{20}(t) & x_{20}(t)=f_{20}\left(\left(u(t), \boldsymbol{p}_{20}\right), G_{U 20}(t)\right) & G_{U 20}(t) \\
U_{21} & S_{21}(t) & \boldsymbol{p}_{21}\left(\psi_{1}, \psi_{2}, \cdots, \psi_{n}\right) & T_{21}(t) & L_{21}(t) & x_{21}(t)=f_{21}\left(\left(u(t), \boldsymbol{p}_{21}\right), G_{U 21}(t)\right) & G_{U 21}(t) \\
& & \vdots & & & \\
U_{2 t} & S_{2 t}(t) & \boldsymbol{p}_{2 t}\left(\psi_{1}, \psi_{2}, \cdots, \psi_{n}\right) & T_{2 t}(t) & L_{2 t}(t) & x_{2 t}(t)=f_{2 t}\left(\left(u(t), \boldsymbol{p}_{2 t}\right), G_{U 2 t}(t)\right) & G_{U 2 t}(t)
\end{array}\right],
\end{aligned}
$$

Table 1. Classification of equational-type error matrix equation.

\begin{tabular}{cccc}
\hline Equation & Type 1 & Type 2 & Meaning of operator \\
\hline 1 & $A X=B$ & $X A=B$ & Generic matrix multiplication \\
2 & $A \bullet X=B$ & $X \bullet A=B$ & Good \\
3 & $A \wedge X=B$ & $X \wedge A=B$ & Bad \\
4 & $A \vee X=B$ & $X \vee A=B$ & Or \\
5 & $A \wedge X=B$ & $X \wedge A=B$ & And \\
\hline
\end{tabular}

Table 2. Classification of containing-type error matrix equation.

\begin{tabular}{cccc}
\hline Equation & Type 1 & Type 2 & Meaning of operator \\
\hline 1 & $A X \supseteq B$ & $X A \supseteq B$ & Generic matrix multiplication \\
2 & $A \bullet X \supseteq B$ & $X \bullet A \supseteq B$ & Good \\
3 & $A \wedge X \supseteq B$ & $X \boldsymbol{\Delta}$ Bad \\
4 & $A \vee X \supseteq B$ & $X \vee A \supseteq B$ & Or \\
5 & $A \wedge X \supseteq B$ & $X \wedge A \supseteq B$ & And \\
\hline
\end{tabular}




$$
\begin{aligned}
& B=\left[\begin{array}{cccc}
\left(b_{11}, y_{11}\right) & \left(b_{12}, y_{12}\right) & \cdots & \left(b_{1 m 1}, y_{1 m 1}\right) \\
\left(b_{21}, y_{21}\right) & \left(b_{22}, y_{22}\right) & \cdots & \left(b_{2 m 1}, y_{2 m 1}\right) \\
\vdots & \vdots & & \vdots \\
\left(b_{m 21}, y_{m 21}\right) & \left(b_{m 22}, y_{m 22}\right) & \cdots & \left(b_{m 2 m 1}, y_{m 2 m 1}\right)
\end{array}\right] \\
& =\left[\begin{array}{ccccc}
V_{201} & S_{V 201}(t) & \boldsymbol{p}_{V 201}\left(\psi_{1}, \psi_{2}, \cdots, \psi_{n}\right) & T_{V 201}(t) & L_{V 201}(t) \\
\cdots V_{21 j} & S_{V 21 j}(t) & \boldsymbol{p}_{V 21 j}\left(\psi_{1}, \psi_{2}, \cdots, \psi_{n}\right) & T_{V 21 j}(t) & L_{V 21 j}(t) \\
& & & \cdots \\
\cdots V_{2 m 2 m 1} & S_{V 2 m 2 m 1}(t) & \boldsymbol{p}_{V 2 m 2 m 1}\left(\psi_{1}, \psi_{2}, \cdots, \psi_{n}\right) & T_{V 2 m 2 m 1}(t) & L_{V 2 m 2 m 1}(t)
\end{array}\right. \\
& y_{V 201}(t)=f_{V 201}\left(\left(v(t), \boldsymbol{p}_{V 201}\right), G_{V 201}(t)\right) \quad G_{V 201}(t) \cdots \\
& y_{V 21 j}(t)=f_{V 21 j}\left(\left(v(t), \boldsymbol{p}_{V 21 j}\right), G_{V 21 j}(t)\right) \quad G_{V 21 j}(t) \cdots \\
& \left.y_{V 2 m 2 m 1}(t)=f_{V 2 m 2 m 1}\left(\left(v(t), \boldsymbol{p}_{V 2 m 2 m 1}\right), G_{V 2 m 2 m 1}(t)\right) \quad G_{V 2 m 2 m 1}(t)\right]
\end{aligned}
$$

\section{Definition 3.1. Suppose}

$$
X A^{\prime}=\left[\begin{array}{cccc}
\left(w_{11}, z_{11}\right) & \left(w_{12}, z_{12}\right) & \ldots & \left(w_{1 m 1}, z_{1 m 1}\right) \\
\left(w_{21}, z_{21}\right) & \left(w_{22}, z_{22}\right) & \cdots & \left(w_{2 m 1}, z_{2 m 1}\right) \\
\vdots & \vdots & & \vdots \\
\left(w_{m 21}, z_{m 21}\right) & \left(w_{m 22}, z_{m 22}\right) & \cdots & \left(w_{m 2 m 1}, z_{m 2 m 1}\right)
\end{array}\right],
$$

One element of the matrix

$\left(w_{i j}, z_{i j}\right)=\left(U_{1 i x} \wedge U_{2 j} S_{1 i x}(t) \wedge S_{2 j}(t) \boldsymbol{p}_{1 i x} \wedge \boldsymbol{p}_{2 j} T_{1 i x}(t) \wedge T_{2 j}(t) L_{1 i x}(t) \wedge L_{2 j}(t) x_{1 i x}(t) \wedge y_{2 j}(t) G_{U 1 i x}(t) \wedge G_{U 2 j}(t)\right)$

Therefore

$$
\begin{aligned}
& {\left[\begin{array}{ccccccc}
U_{10 X} \wedge U_{20} & S_{10 X}(t) \wedge S_{20}(t) & \boldsymbol{p}_{10 X} \wedge \boldsymbol{p}_{20}(t) & T_{10 X}(t) \wedge T_{20}(t) & L_{10 X}(t) \wedge L_{20}(t) & x_{10 X}(t) \wedge x_{20}(t) & G_{U 10 X}(t) \wedge G_{U 20}(t) \cdots \\
\cdots U_{11 X} \wedge U_{21} & S_{11 X}(t) \wedge S_{21}(t) & \boldsymbol{p}_{11 X} \wedge \boldsymbol{p}_{21}(t) & T_{11 X}(t) \wedge T_{21}(t) & L_{11 X}(t) \wedge L_{21}(t) & x_{11 X}(t) \wedge x_{21}(t) & G_{U 11 X}(t) \wedge G_{U 21}(t) \cdots \\
& & \vdots & & & \\
& & & & & & \\
\cdots U_{1 t X} \wedge U_{2 t} & S_{1 t X}(t) \wedge S_{2 t}(t) & \boldsymbol{p}_{1 t X} \wedge \boldsymbol{p}_{2 t}(t) & T_{1 t X}(t) \wedge T_{2 t}(t) & L_{1 t X}(t) \wedge L_{2 t}(t) & x_{1 t X}(t) \wedge x_{2 t}(t) & G_{U 1 t X}(t) \wedge G_{U 2 t}(t)
\end{array}\right]} \\
& =\left[\begin{array}{ccccc}
V_{201} & S_{V 201}(t) & \boldsymbol{p}_{V 201}\left(\psi_{1}, \psi_{2}, \cdots, \psi_{n}\right) & T_{V 201}(t) & L_{V 201}(t) \\
\cdots V_{21 j} & S_{V 21 j}(t) & \boldsymbol{p}_{V 21 j}\left(\psi_{1}, \psi_{2}, \cdots, \psi_{n}\right) & T_{V 21 j}(t) & L_{V 21 j}(t) \\
& & \cdots & \\
\cdots V_{2 m 2 m 1} & S_{V 2 m 2 m 1}(t) & \boldsymbol{p}_{V 2 m 2 m 1}\left(\psi_{1}, \psi_{2}, \cdots, \psi_{n}\right) & T_{V 2 m 2 m 1}(t) & L_{V 2 m 2 m 1}(t)
\end{array}\right. \\
& \left.y_{V 201}(t)=f_{V 201}\left(\left(v(t), \boldsymbol{p}_{V 201}\right), G_{V 201}(t)\right) \quad G_{V 201}(t) \cdots\right] \\
& y_{V 21 j}(t)=f_{V 21 j}\left(\left(v(t), \boldsymbol{p}_{V 21 j}\right), G_{V 21 j}(t)\right) \quad G_{V 21 j}(t) \cdots \\
& \left.y_{V 2 m 2 m 1}(t)=f_{V 2 m 2 m 1}\left(\left(v(t), \boldsymbol{p}_{V 2 m 2 m 1}\right), G_{V 2 m 2 m 1}(t)\right) G_{V 2 m 2 m 1}(t)\right]
\end{aligned}
$$

According to the definition of matrix equivalent: $\left(w_{i j}, z_{i j}\right) \supseteq\left(b_{i j}, y_{i j}\right)$.

So

$$
\begin{aligned}
& \left(U_{1 i X} \wedge U_{2 j} S_{1 i X}(t) \wedge S_{2 j}(t) \boldsymbol{p}_{1 i X}\left(\psi_{1}, \psi_{2}, \cdots, \psi_{n}\right) \wedge \boldsymbol{p}_{2 j} T_{1 i X}(t) \wedge T_{2 j}(t)\right. \\
& \left.L_{1 i X}(t) \wedge L_{2 j}(t) x_{1 i X}(t)=f_{1 i X}\left(\left(u(t), p_{1 i X}\right), G_{U l i X}(t)\right) \wedge x_{2 j}(t) G_{U 1 i X}(t) \wedge G_{U 2 j}(t)\right) \supseteq\left(b_{i j}, y_{i j}\right),
\end{aligned}
$$


That is

$$
\begin{aligned}
& \left(U_{1 i X} \wedge U_{2 j} S_{1 i X}(t) \wedge S_{2 j}(t) \boldsymbol{p}_{1 i X}\left(\psi_{1}, \psi_{2}, \cdots, \psi_{n}\right) \wedge \boldsymbol{p}_{2 j} T_{1 i X}(t) \wedge T_{2 j}(t)\right. \\
& \left.L_{1 i X}(t) \wedge L_{2 j}(t) x_{1 i X}(t)=f_{1 i X}\left(\left(u(t), \boldsymbol{p}_{1 i X}\right), G_{U 1 i X}(t)\right) \wedge x_{2 j}(t) G_{U 1 X X}(t) \wedge G_{U 2 j}(t)\right) \\
& \supseteq\left(V_{2 i j} S_{V 2 i j}(t) \boldsymbol{p}_{V 2 i j} T_{V 2 i j}(t) L_{V 2 i j}(t) y_{V 2 i j}(t) G_{V 2 i j}(t)\right) .
\end{aligned}
$$

i.e., according to the corresponding factors in these two matrixes, the equations below are obtained:

$$
\begin{aligned}
& U_{10 X} \wedge U_{20} \supseteq V_{V 20}, \\
& S_{10 X}(t) \wedge S_{20}(t) \supseteq S_{V 20}(t), \\
& \boldsymbol{p}_{10 X}\left(\psi_{1}, \psi_{2}, \cdots, \psi_{n}\right) \wedge \boldsymbol{p}_{20} \supseteq \boldsymbol{p}_{V 20}\left(\psi_{1}, \psi_{2}, \cdots, \psi_{n}\right), \\
& T_{10 X}(t) \wedge T_{20}(t) \supseteq T_{V 20}(t), \\
& L_{10 X}(t) \wedge L_{20}(t) \supseteq L_{V 20}(t), \\
& x_{10 X}(t) \wedge x_{20}(t) \geq y_{V 20}(t), \\
& G_{U 10 X}(t) \wedge G_{U 20}(t) \supseteq G_{V 20}(t) ; \\
& \vdots \\
& U_{1 i X} \wedge U_{2 j} \supseteq V_{V 2 j}, \\
& S_{1 i X}(t) \wedge S_{2 j}(t) \supseteq S_{V 2 j}(t), \\
& \boldsymbol{p}_{1 i X}\left(\psi_{1}, \psi_{2}, \cdots, \psi_{n}\right) \wedge \boldsymbol{p}_{2 j} \supseteq \boldsymbol{p}_{V 2 j}\left(\psi_{1}, \psi_{2}, \cdots, \psi_{n}\right), \\
& T_{1 i X}(t) \wedge T_{2 j}(t) \supseteq T_{V 2 j}(t), \\
& L_{1 i X}(t) \wedge L_{2 j}(t) \supseteq L_{V 2 j}(t), \\
& x_{1 i X}(t) \wedge x_{2 j}(t) \geq y_{V 2 j}(t), \\
& G_{U 1 i X}(t) \wedge G_{U 2 j}(t) \supseteq G_{V 2 j}(t) .
\end{aligned}
$$

If both sides of the equation are all sets, operator " $\wedge$ "means the operation of intersection. If both sides of the equation are values, operator " $\wedge$ "means the opera- tion of minimization.

In fact, the equation sets above are not irrelevant. After calculation, the factors of matrix can compose a complete proposition. This can be defined as

$$
\begin{aligned}
& \left(U_{1 i X} \wedge U_{2 j}\right) h_{1}\left(S_{1 i X}(t) \wedge S_{2 j}(t)\right) \\
& h_{2}\left(\boldsymbol{p}_{1 i X}\left(\psi_{1}, \psi_{2}, \cdots \psi_{n}\right) \wedge \boldsymbol{p}_{2 j}\right) h_{3}\left(T_{1 i X}(t) \wedge T_{2 j}(t)\right) \\
& h_{4}\left(L_{1 i X}(t) \wedge L_{2 j}(t)\right) h_{5}\left(x_{1 i X}(t) \wedge x_{2 j}(t)\right) \\
& h_{6}\left(G_{U 11 X}(t) \wedge G_{U 2 j}(t)\right) .
\end{aligned}
$$

" $h_{i}, i=1,2, \cdots, 6$ " means a complete matrix element composition can be composed after calculating. The methods of composing are decided according to the specific situation. One way is the multiplication of $m \times 7$ order error matrix, from which is composed a new error set or error logical proposition by using each parameter from calculation to be the corresponding parameter.

Theorem 1. A necessary and sufficient condition of solving error matrix equation $X A^{\prime} \supseteq B$ is that equation $X_{i} A^{\prime} \supseteq B_{i}, i=(1,2, \cdots, m 2)$ has a solution.

Proof: if $X A^{\prime}=\mathrm{B}$ has a solution, according to the equivalent equations $X A^{\prime} \supseteq B$ and $X_{i} A^{\prime} \supseteq B_{i}, i=(1,2$, $\cdots, m 2)$, the equation $X_{i} A^{\prime} \supseteq B_{i}, i=(1,2, \cdots, m 2)$ should have a solution first; Vice versa, if $X_{i} A^{\prime} \supseteq B_{i}, i=(1,2$, $\cdots, m 2$ ) has a solution, we also can know $X A^{\prime} \supseteq B$ has a solution by the same principle.

So, we can discuss the solution of equation $X A^{\prime} \supseteq B$, the same as when solving $\quad X_{i} A^{\prime} \supseteq B_{i}, i=(1,2, \cdots, m 2)$.

With respect to $X_{i} A^{\prime} \supseteq B_{i}$,

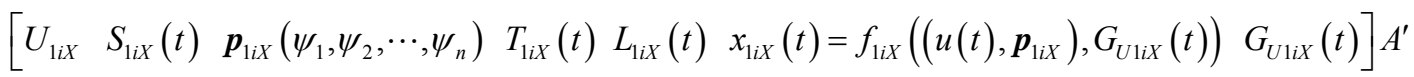

$$
\begin{aligned}
& =\left[\left(U_{1 i X} \wedge U_{20}\right) \vee\left(S_{1 i X}(t) \wedge S_{20}(t)\right) \vee\left(\boldsymbol{p}_{1 i X}\left(\psi_{1}, \psi_{2}, \cdots \psi_{n}\right) \wedge \boldsymbol{p}_{20}\right) \vee\left(T_{1 i X}(t) \wedge T_{20}(t)\right)\right. \\
& \vee\left(L_{1 i X}(t) \wedge L_{20}(t)\right) \vee\left(x_{1 i X}(t) \wedge x_{20}(t)\right) \vee\left(G_{U 1 X X}(t) \wedge G_{U 20}(t)\right) \\
& \left(U_{1 i X} \wedge U_{2 j}\right) \vee\left(S_{1 i X}(t) \wedge S_{2 j}(t)\right) \vee\left(\boldsymbol{p}_{1 i X}\left(\psi_{1}, \psi_{2}, \cdots \psi_{n}\right) \wedge \boldsymbol{p}_{2 j}\right) \vee\left(T_{1 i X}(t) \wedge T_{2 j}(t)\right) \\
& \vee\left(L_{1 i X}(t) \wedge L_{2 j}(t)\right) \vee\left(x_{1 i X}(t) \wedge x_{2 j}(t)\right) \vee\left(G_{U 1 i X}(t) \wedge G_{U 2 j}(t)\right) \\
& {\left[\left(U_{1 i X} \wedge U_{2 m 1}\right) \vee\left(S_{1 i X}(t) \wedge S_{2 m 1}(t)\right) \vee\left(\boldsymbol{p}_{1 i X}\left(\psi_{1}, \psi_{2}, \cdots \psi_{n}\right) \wedge \boldsymbol{p}_{2 m 1}\right) \vee\left(T_{1 i X}(t) \wedge T_{2 m 1}(t)\right)\right.} \\
& \left.\vee\left(L_{1 i X}(t) \wedge L_{2 m 1}(t)\right) \vee\left(x_{1 i X}(t) \wedge x_{2 m 1}(t)\right) \vee\left(G_{U 1 i X}(t) \wedge G_{U 2 m 1}(t)\right)\right] \supseteq\left[\left(b_{i 1}, y_{i 1}\right) \quad\left(b_{i 2}, y_{i 2}\right) \cdots\left(b_{i m 1}, y_{i m 1}\right)\right] \text {. }
\end{aligned}
$$

i.e. 
$\left(U_{1 i X} \wedge U_{20}\right) \vee\left(S_{1 i X}(t) \wedge S_{20}(t)\right) \vee\left(\boldsymbol{p}_{1 i X}\left(\psi_{1}, \psi_{2}, \cdots \psi_{n}\right) \wedge \boldsymbol{p}_{20}\right) \vee\left(T_{1 i X}(t) \wedge T_{20}(t)\right) \vee\left(L_{1 i X}(t) \wedge L_{20}(t)\right) \vee\left(x_{1 i X}(t) \wedge x_{20}(t)\right)$ $\left.\vee\left(G_{U 11 X}(t) \wedge G_{U 20}(t)\right) \supseteq\left(\begin{array}{llllll}V_{20} & S_{V 20}(t) & \boldsymbol{p}_{V 20}\left(\psi_{1}, \psi_{2}, \cdots \psi_{n}\right.\end{array}\right) T_{V 20}(t) \quad L_{V 20}(t) \quad y_{V 20}(t) \quad G_{V 20}(t)\right) ;$

$\left(U_{1 i X} \wedge U_{2 j}\right) \vee\left(S_{1 i X}(t) \wedge S_{2 j}(t)\right) \vee\left(\boldsymbol{p}_{1 i X}\left(\psi_{1}, \psi_{2}, \cdots \psi_{n}\right) \wedge \boldsymbol{p}_{2 j}\right) \vee\left(T_{1 i X}(t) \wedge T_{2 j}(t)\right) \vee\left(L_{1 i X}(t) \wedge L_{2 j}(t)\right) \vee\left(x_{1 i X}(t) \wedge x_{2 j}(t)\right)$ $\vee\left(G_{U 11 X}(t) \wedge G_{U 2 j}(t)\right) \supseteq\left(V_{2 j} \quad S_{V 2 j}(t) \quad \boldsymbol{p}_{V 2 j}\left(\psi_{1}, \psi_{2}, \cdots \psi_{n}\right) T_{V 2 j}(t) L_{V 2 j}(t) \quad y_{V 2 j}(t) G_{V 2 j}(t)\right)$;

$\left(U_{1 t X} \wedge U_{2 m 1}\right) \vee\left(S_{1 t X}(t) \wedge S_{2 m 1}(t)\right) \vee\left(\boldsymbol{p}_{1 t X}\left(\psi_{1}, \psi_{2}, \cdots \psi_{n}\right) \wedge \boldsymbol{p}_{2 m 1}\right) \vee\left(T_{1 t X}(t) \wedge T_{2 m 1}(t)\right) \vee\left(L_{1 t X}(t) \wedge L_{2 m 1}(t)\right)$ $\vee\left(x_{1 t X}(t) \wedge x_{2 m 1}(t)\right) \vee\left(G_{U 1 t X}(t) \wedge G_{U 2 m 1}(t)\right) \supseteq\left(\begin{array}{lllllll}V_{2 t} & S_{V 2 t}(t) & \boldsymbol{p}_{V 2 t}\left(\psi_{1}, \psi_{2}, \cdots \psi_{n}\right) & T_{V 2 t}(t) & L_{V 2 t}(t) & y_{V 2 t}(t) & \left.G_{V 2 t}(t)\right) .\end{array}\right.$

There are equations:

$\left(U_{1 i X} \wedge U_{20}\right) \supseteq V_{V 20}$,

$\left(S_{1 i X}(t) \wedge S_{20}(t)\right) \supseteq S_{V 20}(t)$,

$\left(\boldsymbol{p}_{1 i X}\left(\psi_{1}, \psi_{2}, \cdots, \psi_{n}\right) \wedge \boldsymbol{p}_{20}\right) \supseteq \boldsymbol{p}_{V 20}\left(\psi_{1}, \psi_{2}, \cdots, \psi_{n}\right)$,

$\left(T_{1 i X}(t) \wedge T_{20}(t)\right) \supseteq T_{V 20}(t)$,

$\left(L_{1 i X}(t) \wedge L_{20}(t)\right) \supseteq L_{V 20}(t)$,

$\left(x_{1 i X}(t) \wedge x_{20}(t)\right) \geq y_{V 20}(t)$,

$\left(G_{U 1 X X}(t) \wedge G_{U 20}(t)\right) \supseteq G_{V 20}(t) ;$

:

$\left(U_{1 i X} \wedge U_{2 j}\right) \supseteq V_{V 2 j}$,

$\left(S_{1 i X}(t) \wedge S_{2 j}(t)\right) \supseteq S_{V 2 j}(t)$,

$\left(\boldsymbol{p}_{1 i X}\left(\psi_{1}, \psi_{2}, \cdots, \psi_{n}\right) \wedge \boldsymbol{p}_{2 j}\right) \supseteq \boldsymbol{p}_{V 2 j}\left(\psi_{1}, \psi_{2}, \cdots, \psi_{n}\right)$,

$\left(T_{1 i X}(t) \wedge T_{2 j}(t)\right) \supseteq T_{V 2 j}(t)$,

$\left(L_{1 i X}(t) \wedge L_{2 j}(t)\right) \supseteq L_{V 2 j}(t)$,

$\left(x_{1 i X}(t) \wedge x_{2 j}(t)\right) \geq y_{V 2 j}(t)$,

$\left(G_{U 1 i X}(t) \wedge G_{U 2 j}(t)\right) \supseteq G_{V 2 j}(t) ;$$$
\text { ! }
$$

$\left(U_{1 t X} \wedge U_{2 m 1}\right) \supseteq V_{V 2 t}$,

$\left(S_{1 i X}(t) \wedge S_{2 m 1}(t)\right) \supseteq S_{V 2 t}(t)$,

$\left(\boldsymbol{p}_{1 i X}\left(\psi_{1}, \psi_{2}, \cdots, \psi_{n}\right) \wedge \boldsymbol{p}_{2 m 1}\right) \supseteq \boldsymbol{p}_{V 2 t}\left(\psi_{1}, \psi_{2}, \cdots, \psi_{n}\right)$,

$\left(T_{1 i X}(t) \wedge T_{2 m 1}(t)\right) \supseteq T_{V 2 t}(t)$,

$\left(L_{1 i X}(t) \wedge L_{2 m 1}(t)\right) \supseteq L_{V 2 t}(t)$,

$\left(x_{1 i X}(t) \wedge x_{2 m 1}(t)\right) \geq y_{V 2 t}(t)$,
$\left(G_{U 1 i X}(t) \wedge G_{U 2 m 1}(t)\right) \supseteq G_{V 2 t}(t)$.

Theorem 2. Necessary and sufficient condition of solving error matrix equation $X_{i} A^{\prime} \supseteq B_{i}$ is:

$$
\begin{aligned}
& U_{20} \supseteq V_{V 20}, \\
& S_{20}(t) \supseteq S_{V 20}(t), \\
& \boldsymbol{p}_{20} \supseteq \boldsymbol{p}_{V 20}\left(\psi_{1}, \psi_{2}, \cdots, \psi_{n}\right), \\
& T_{20}(t) \supseteq T_{V 20}(t), \\
& L_{20}(t) \supseteq L_{V 20}(t), \\
& x_{20}(t) \geq y_{V 20}(t), \\
& G_{U 20}(t) \supseteq G_{V 20}(t) ; \\
& \vdots \\
& U_{2 j} \supseteq V_{V 2 j}, \\
& S_{2 j}(t) \supseteq S_{V 2 j}(t), \\
& \boldsymbol{p}_{2 j} \supseteq \boldsymbol{p}_{V 2 j}\left(\psi_{1}, \psi_{2}, \cdots, \psi_{n}\right), \\
& T_{2 j}(t) \supseteq T_{V 2 j}(t), \\
& L_{2 j}(t) \supseteq L_{V 2 j}(t), \\
& x_{2 j}(t) \geq y_{V 2 j}(t), \\
& G_{U 2 j}(t) \supseteq G_{V 2 j}(t) ; \\
& \vdots \\
& U_{2 m 1} \supseteq V_{V 2 t}, \\
& S_{2 m 1}(t) \supseteq S_{V 2 t}(t), \\
& \boldsymbol{p}_{2 m 1} \supseteq \boldsymbol{p}_{V 2 t}\left(\psi_{1}, \psi_{2}, \cdots, \psi_{n}\right), \\
& T_{2 m 1}(t) \supseteq T_{V 2 t}(t), \\
& L_{2 m 1}(t) \supseteq L_{V 2 t}(t), \\
& x_{2 m 1}(t) \geq y_{V 2 t}(t), \\
&
\end{aligned}
$$




$$
G_{U 2 m 1}(t) \supseteq G_{V 2 t}(t) .
$$

Proof of necessity: If one of above conditions is not satisfied, for example, condition $S_{2 j}(t) \supseteq S_{V 2 j}(t)$, is not satisfied, then in equation $\left(S_{1 i X}(t) \wedge S_{2 j}(t)\right) \supseteq S_{V 2 j}(t)$, no matter what the value of $S_{1 i X}(t)$ is, $\left(S_{1 i X}(t) \wedge S_{2 j}(t)\right) \supseteq S_{V 2 j}(t)$ cannot be calculated.

Proof of sufficiency: The condition $A \supseteq B_{i}$ is satisfied in error matrix equation $X_{i} A^{\prime} \supseteq B_{i}$, so the element of $X_{i}$, which corresponds to element $A$, take union of set. i.e.,

$$
\begin{aligned}
& U_{1 i X}=U_{20} \cup U_{21} \cup \cdots \cup U_{2 j} \cup \cdots \cup U_{2 t}, \\
& S_{1 i X}=S_{20} \cup S_{21} \cup \cdots \cup S_{2 j} \cup \cdots \cup S_{2 t}, \\
& \boldsymbol{p}_{1 i X}\left(\psi_{1}, \psi_{2}, \cdots \psi_{n}\right)=\boldsymbol{p}_{20} \cup \boldsymbol{p}_{21} \cup \cdots \cup \boldsymbol{p}_{2 j} \cup \cdots \cup \boldsymbol{p}_{2 t}, \\
& T_{1 i X}=T_{20} \cup T_{21} \cup \cdots \cup T_{2 j} \cup \cdots \cup T_{2 t},
\end{aligned}
$$$$
a_{11}=\left(U_{201} S_{201}(t) \boldsymbol{p}_{201}\left(\psi_{1}, \psi_{2}, \cdots, \psi_{n}\right) T_{201}(t) L_{201}(t) x_{201}(t)=f_{201}\left(\left(u(t), \boldsymbol{p}_{201}\right), G_{U 201}(t)\right) G_{U 201}(t)\right)
$$

\section{Application Example of Error Matrix Equation}

For example: suppose $A^{\prime}=\left[\begin{array}{ll}a_{11} & a_{12} \\ a_{21} & a_{22}\end{array}\right]$,

Then discussing the whole solution of equations

After solving $X\left(x_{1}, x_{2}, \cdots x_{n}\right)$ of $X_{i} A^{\prime} \supseteq B_{i}$, by taking the intersection of $X, \mathrm{~kg}, r w$ and $x q$, we can acquire

ing the intersection of $X, k \mathrm{~g}, r w$ and $x q$, we can acquire
$X\left(x_{1}^{\prime}, x_{2}^{\prime}, \cdots x_{n}^{\prime}\right) \in X$.

$$
\begin{aligned}
& L_{1 i X}=L_{20} \cup L_{21} \cup \cdots \cup L_{2 j} \cup \cdots \cup L_{2 t}, \\
& x_{1 i X}(t)=x_{20}(x) \cup x_{21}(x) \cup \cdots \cup x_{2 j}(x) \cup \cdots \cup x_{2 t}(t), \\
& G_{U 1 i X}(t) \\
& =G_{U 20}(x) \cup G_{U 21}(x) \cup \cdots \cup G_{U 2 j}(x) \cup \cdots \cup G_{U 2 t}(t) . \\
& \text { Q.ed. }
\end{aligned}
$$
$X_{i} A^{\prime} \supseteq B_{i}$ and $X A^{\prime} \supseteq B$

$$
\begin{aligned}
& a_{12}=\left(\begin{array}{llll}
U_{202} & S_{202}(t) & \boldsymbol{p}_{202}\left(\psi_{1}, \psi_{2}, \cdots, \psi_{n}\right) & T_{202}(t) L_{202}(t) x_{202}(t)=f_{202}\left(\left(u(t), \boldsymbol{p}_{202}\right), G_{U 202}(t)\right) G_{U 202}(t)
\end{array}\right), \\
& a_{21}=\left(\begin{array}{lllll}
U_{211} & S_{211}(t) & \boldsymbol{p}_{211}\left(\psi_{1}, \psi_{2}, \cdots, \psi_{n}\right) & T_{211}(t) L_{211}(t) x_{211}(t)=f_{211}\left(\left(u(t), \boldsymbol{p}_{211}\right), G_{U 211}(t)\right) G_{U 211}(t)
\end{array}\right), \\
& a_{22}=\left(\begin{array}{llll}
U_{212} & S_{212}(t) & \boldsymbol{p}_{212}\left(\psi_{1}, \psi_{2}, \cdots, \psi_{n}\right) & \left.T_{212}(t) L_{212}(t) x_{212}(t)=f_{212}\left(\left(u(t), \boldsymbol{p}_{212}\right), G_{U 212}(t)\right) G_{U 212}(t)\right)
\end{array}\right)
\end{aligned}
$$

Each element included in $a_{11}, a_{12}, a_{21}, a_{22}$ is A set type. For instance, an element of discussing domain $U_{201}$ has several elements under it. So $U_{201}$ can be expressed as $U_{201}=\left\{u_{201}, u_{202}, \cdots, u_{20 n}\right\}$. Thus, we could express all the elements as follows:

$$
\begin{aligned}
& U_{201}=\left\{u_{201}, u_{202}, \cdots, u_{20 n}\right\}, \\
& S_{201}(t)=\left\{s_{201}, s_{202}, \cdots, s_{20 n}\right\}, \\
& \boldsymbol{p}_{201}\left(\psi_{1}, \psi_{2}, \cdots, \psi_{n}\right)=\left\{\boldsymbol{p}_{201}, \boldsymbol{p}_{202}, \cdots, \boldsymbol{p}_{20 n}\right\}, \\
& T_{201}(t)=\left\{t_{201}, t_{202}, \cdots, t_{20 n}\right\}, \\
& L_{201}(t)=\left\{l_{201}, l_{202}, \cdots, l_{20 n}\right\}, \\
& x_{201}(t)=\left\{x_{201}, x_{202}, \cdots, x_{20 n}\right\}, \\
& G_{U 201}(t)=\left\{g_{201}, g_{202}, \cdots, g_{20 n}\right\}, \\
& n=11 ; \\
& U_{202}=\left\{u_{201}, u_{202}, \cdots, u_{20 n}\right\}, \\
& S_{202}(t)=\left\{s_{201}, s_{202}, \cdots, s_{20 n}\right\}, \\
& \boldsymbol{p}_{202}\left(\psi_{1}, \psi_{2}, \cdots, \psi_{n}\right)=\left\{\boldsymbol{p}_{201}, \boldsymbol{p}_{202}, \cdots, \boldsymbol{p}_{20 n}\right\}, \\
& T_{202}(t)=\left\{t_{201}, t_{202}, \cdots, t_{20 n}\right\},
\end{aligned}
$$$$
\begin{aligned}
& L_{202}(t)=\left\{l_{201}, l_{202}, \cdots, l_{20 n}\right\}, \\
& x_{202}(t)=\left\{x_{201}, x_{202}, \cdots, x_{20 n}\right\}, \\
& G_{202}(t)=\left\{g_{201}, g_{202}, \cdots, g_{20 n}\right\}, \\
& n=9 ; \\
& U_{211}=\left\{u_{211}, u_{212}, \cdots, u_{21 n}\right\}, \\
& S_{211}(t)=\left\{s_{211}, s_{212}, \cdots, s_{21 n}\right\}, \\
& \boldsymbol{p}_{211}\left(\psi_{1}, \psi_{2}, \cdots, \psi_{n}\right)=\left\{\boldsymbol{p}_{211}, \boldsymbol{p}_{212}, \cdots, \boldsymbol{p}_{21 n}\right\}, \\
& \boldsymbol{p}_{211}\left(\psi_{1}, \psi_{2}, \cdots, \psi_{n}\right)=\left\{\boldsymbol{p}_{211}, \boldsymbol{p}_{212}, \cdots, \boldsymbol{p}_{21 n}\right\}, \\
& T_{211}(t)=\left\{t_{211}, t_{212}, \cdots, t_{21 n}\right\}, \\
& L_{211}(t)=\left\{l_{211}, l_{212}, \cdots, l_{21 n}\right\}, \\
& x_{211}(t)=\left\{x_{211}, x_{212}, \cdots, x_{21 n}\right\}, \\
& G_{211}(t)=\left\{g_{211}, g_{212}, \cdots, g_{21 n}\right\}, \\
& n=10 ; \\
& U_{212}=\left\{u_{211}, u_{212}, \cdots, u_{21 n}\right\}, \\
& S_{212}(t)=\left\{s_{211}, s_{212}, \cdots, s_{21 n}\right\},
\end{aligned}
$$ 


$$
\begin{array}{ll}
\boldsymbol{p}_{212}\left(\psi_{1}, \psi_{2}, \cdots, \psi_{n}\right)=\left\{\boldsymbol{p}_{211}, \boldsymbol{p}_{212}, \cdots, \boldsymbol{p}_{21 n}\right\}, & x_{212}(t)=\left\{x_{211}, x_{212}, \cdots, x_{21 n}\right\}, \\
T_{212}(t)=\left\{t_{211}, t_{212}, \cdots, t_{21 n}\right\}, & G_{212}(t)=\left\{g_{211}, g_{212}, \cdots, g_{21 n}\right\}, \\
L_{212}(t)=\left\{l_{211}, l_{212}, \cdots, l_{21 n}\right\}, & n=15 ;
\end{array}
$$

Suppose $X=\left(\begin{array}{ll}x_{1} & x_{2}\end{array}\right)$,

$$
\begin{aligned}
& x_{1}=\left(U_{10 X} S_{10 X}(t) \boldsymbol{p}_{10 X}\left(\psi_{1}, \psi_{2}, \cdots, \psi_{n}\right) T_{10 X}(t) L_{10 X}(t) x_{10 X}(t)=f_{10 X}\left(\left(u(t), \boldsymbol{p}_{201}\right), G_{U 10 X}(t)\right) G_{U 10 X}(t)\right), \\
& x_{2}=\left(U_{11 X} S_{11 X}(t) \boldsymbol{p}_{11 X}\left(\psi_{1}, \psi_{2}, \cdots, \psi_{n}\right) T_{11 X}(t) L_{11 X}(t) x_{11 X}(t)=f_{11 X}\left(\left(u(t), \boldsymbol{p}_{201}\right), G_{U 11 X}(t)\right) G_{U 11 X}(t)\right) \text {, }
\end{aligned}
$$

and $B^{\prime}=\left[\begin{array}{ll}b_{11} & b_{12} \\ b_{21} & b_{22}\end{array}\right]$,

$$
\begin{aligned}
& b_{11}=\left(V_{201} S_{V 201}(t) \boldsymbol{p}_{V 201}\left(\psi_{1}, \psi_{2}, \cdots, \psi_{n}\right) T_{V 201}(t) L_{V 201}(t) y_{V 201}(t)=f_{V 201}\left(\left(u(t), \boldsymbol{p}_{V 201}\right), G_{V 201}(t)\right) G_{V 201}(t)\right) \text {, } \\
& b_{12}=\left(V_{202} S_{V 202}(t) \boldsymbol{p}_{V 202}\left(\psi_{1}, \psi_{2}, \cdots, \psi_{n}\right) T_{V 202}(t) L_{V 202}(t) y_{V 202}(t)=f_{V 202}\left(\left(u(t), \boldsymbol{p}_{V 202}\right), G_{V 202}(t)\right) G_{V 202}(t)\right) \text {, } \\
& b_{21}=\left(V_{211} S_{V 211}(t) \boldsymbol{p}_{V 211}\left(\psi_{1}, \psi_{2}, \cdots, \psi_{n}\right) T_{V 211}(t) L_{V 211}(t) y_{V 211}(t)=f_{V 211}\left(\left(u(t), \boldsymbol{p}_{V 211}\right), G_{V 211}(t)\right) G_{V 211}(t)\right), \\
& b_{22}=\left(V_{212} S_{V 212}(t) \boldsymbol{p}_{V 212}\left(\psi_{1}, \psi_{2}, \cdots, \psi_{n}\right) T_{V 212}(t) L_{V 212}(t) y_{V 212}(t)=f_{V 212}\left(\left(u(t), \boldsymbol{p}_{V 212}\right), G_{V 212}(t)\right) G_{V 212}(t)\right) \text {. }
\end{aligned}
$$

The representation of all the elements of $b_{11}, b_{12}, b_{21}$, $b_{22}$ is the same as $a_{11}, a_{12}, a_{21}, a_{22}$. So they can be expressed as follows:

$$
\begin{aligned}
& V_{201}=\left\{u_{201}, u_{202}, \cdots, u_{20 k}\right\}, \\
& S_{V 201}(t)=\left\{s_{201}, s_{202}, \cdots, s_{20 k}\right\}, \\
& \boldsymbol{p}_{V 201}\left(\psi_{1}, \psi_{2}, \cdots, \psi_{n}\right)=\left\{\boldsymbol{p}_{201}, \boldsymbol{p}_{202}, \cdots, \boldsymbol{p}_{20 k}\right\}, \\
& T_{V 201}(t)=\left\{t_{201}, t_{202}, \cdots, t_{20 k}\right\}, \\
& L_{V 201}(t)=\left\{l_{201}, l_{202}, \cdots, l_{20 k}\right\}, \\
& y_{V 201}(t)=\left\{y_{201}, y_{202}, \cdots, y_{20 k}\right\}, \\
& G_{V 201}(t)=\left\{g_{201}, g_{202}, \cdots, g_{20 k}\right\}, \\
& k=7 ; \\
& V_{202}=\left\{u_{201}, u_{202}, \cdots, u_{20 k}\right\}, \\
& S_{V 202}(t)=\left\{s_{201}, s_{202}, \cdots, s_{20 k}\right\}, \\
& \boldsymbol{p}_{V 202}\left(\psi_{1}, \psi_{2}, \cdots, \psi_{n}\right)=\left\{\boldsymbol{p}_{201}, \boldsymbol{p}_{202}, \cdots, \boldsymbol{p}_{20 k}\right\}, \\
& T_{V 202}(t)=\left\{t_{201}, t_{202}, \cdots, t_{20 k}\right\}, \\
& L_{V 202}(t)=\left\{l_{201}, l_{202}, \cdots, l_{20 k}\right\}, \\
& y_{V 202}(t)=\left\{y_{201}, y_{202}, \cdots, y_{20 k}\right\}, \\
& G_{V 202}(t)=\left\{g_{201}, g_{202}, \cdots, g_{20 k}\right\},
\end{aligned}
$$$$
k=8 ;
$$$$
V_{211}=\left\{u_{211}, u_{212}, \cdots, u_{21 k}\right\} \text {, }
$$$$
S_{V 211}(t)=\left\{s_{211}, s_{212}, \cdots, s_{21 k}\right\},
$$$$
\boldsymbol{p}_{V 211}\left(\psi_{1}, \psi_{2}, \cdots, \psi_{n}\right)=\left\{\boldsymbol{p}_{211}, \boldsymbol{p}_{212}, \cdots, \boldsymbol{p}_{21 k}\right\} \text {, }
$$$$
T_{V 211}(t)=\left\{t_{211}, t_{212}, \cdots, t_{21 k}\right\},
$$$$
L_{V 211}(t)=\left\{l_{211}, l_{212}, \cdots, l_{21 k}\right\},
$$$$
y_{V 211}(t)=\left\{y_{211}, y_{212}, \cdots, y_{21 k}\right\} \text {, }
$$$$
G_{V 211}(t)=\left\{g_{211}, g_{212}, \cdots, g_{21 k}\right\},
$$$$
k=2 \text {; }
$$$$
V_{212}=\left\{u_{211}, u_{212}, \cdots, u_{21 k}\right\} \text {, }
$$$$
S_{V 212}(t)=\left\{s_{211}, s_{212}, \cdots, s_{21 k}\right\} \text {, }
$$$$
\boldsymbol{p}_{V 212}\left(\psi_{1}, \psi_{2}, \cdots, \psi_{n}\right)=\left\{\boldsymbol{p}_{211}, \boldsymbol{p}_{212}, \cdots, \boldsymbol{p}_{21 k}\right\} \text {, }
$$$$
T_{V 212}(t)=\left\{t_{211}, t_{212}, \cdots, t_{21 k}\right\},
$$$$
L_{V 212}(t)=\left\{l_{211}, l_{212}, \cdots, l_{21 k}\right\},
$$$$
y_{V 212}(t)=\left\{y_{211}, y_{212}, \cdots, y_{21 k}\right\} \text {, }
$$$$
G_{V 212}(t)=\left\{g_{211}, g_{212}, \cdots, g_{21 k}\right\} \text {, }
$$$$
k=3 \text {. }
$$

The three constraints of $k g, r w$ and $x q$ are described 
below.

$$
\begin{aligned}
& k g:\left(U_{k g}, S_{k g}(t), \boldsymbol{p}_{k g}\left(\psi_{1}, \psi_{2}, \cdots, \psi_{n}\right),\right. \\
& \left.T_{k g}(t), L_{k g}(t), X_{k g}(t), G_{U}(t)\right) \\
& U_{k g}=\left\{u_{201}, u_{202}, \cdots, u_{20 n}, v_{201}, v_{201}, \cdots, v_{20 j}\right\}, \\
& S_{k g}(t)=\left\{s_{201}, s_{202}, \cdots, s_{20 n}\right\}, \\
& \boldsymbol{p}_{k g}\left(\psi_{1}, \psi_{2}, \cdots, \psi_{n}\right)=\left\{\boldsymbol{p}_{201}, \boldsymbol{p}_{202}, \cdots, \boldsymbol{p}_{20 n}\right\}, \\
& T_{k g}(t)=\left\{t_{201}, t_{202}, \cdots, t_{20 n}\right\}, \\
& L_{k g}(t)=\left\{l_{201}, l_{202}, \cdots, l_{20 n}\right\}, \\
& y_{k g}(t)=\left\{x_{201}, x_{202}, \cdots, x_{20 n}, y_{211}, y_{212}, \cdots, y_{21 j}\right\}, \\
& G_{U}(t)=\left\{g_{201}, g_{202}, \cdots, g_{20 n}\right\}, \\
& n=4, j=5 .
\end{aligned}
$$$$
r w:\left(U_{r w}, S_{r w}(t), \boldsymbol{p}_{r w}\left(\psi_{1}, \psi_{2}, \cdots, \psi_{n}\right),\right.
$$$$
\left.T_{r w}(t), L_{r w}(t), X_{r w}(t), G_{U}(t)\right) .
$$$$
U_{r w}=\left\{u_{201}, u_{202}, \cdots, u_{20 n}\right\},
$$$$
S_{r w}(t)=\left\{s_{201}, s_{202}, \cdots, s_{20 n}\right\},
$$$$
\boldsymbol{p}_{r w}\left(\psi_{1}, \psi_{2}, \cdots, \psi_{n}\right)=\left\{\boldsymbol{p}_{201}, \boldsymbol{p}_{202}, \cdots, \boldsymbol{p}_{20 n}\right\},
$$$$
T_{r w}(t)=\left\{t_{201}, t_{202}, \cdots, t_{20 n}\right\},
$$$$
L_{r w}(t)=\left\{l_{201}, l_{202}, \cdots, l_{20 n}\right\},
$$$$
y_{r w}(t)=\left\{x_{201}, x_{202}, \cdots, x_{20 n}, y_{211}, y_{212}, \cdots, y_{21 j}\right\} \text {, }
$$$$
G_{U}(t)=\left\{g_{201}, g_{202}, \cdots, g_{20 n}\right\},
$$$$
n=10 \text {. }
$$$$
x q:\left(U_{x q}, S_{x q}(t), \boldsymbol{p}_{x q}\left(\psi_{1}, \psi_{2}, \cdots, \psi_{n}\right),\right.
$$$$
\left.T_{x q}(t), L_{x q}(t), X_{x q}(t), G_{U}(t)\right)
$$$$
U_{x q}=\left\{u_{201}, u_{202}, \cdots, u_{20 n}\right\},
$$$$
S_{x q}(t)=\left\{s_{201}, s_{202}, \cdots, s_{20 n}\right\},
$$$$
\boldsymbol{p}_{x q}\left(\psi_{1}, \psi_{2}, \cdots, \psi_{n}\right)=\left\{\boldsymbol{p}_{201}, \boldsymbol{p}_{202}, \cdots, \boldsymbol{p}_{20 n}\right\},
$$$$
T_{x q}(t)=\left\{t_{201}, t_{202}, \cdots, t_{20 n}\right\},
$$$$
L_{x q}(t)=\left\{l_{201}, l_{202}, \cdots, l_{20 n}\right\},
$$$$
y_{x q}(t)=\left\{x_{201}, x_{202}, \cdots, x_{20 n}, y_{211}, y_{212}, \cdots, y_{21 j}\right\},
$$

$G_{U}(t)=\left\{g_{201}, g_{202}, \cdots, g_{20 n}\right\}$,

$n=8$.

By Theorem 2, the solution of $X A^{\prime} \supseteq B$ is:

$$
\begin{aligned}
& U_{10 X}=\left\{u_{201}, u_{202}, \cdots, u_{20 n}\right\}, \\
& S_{10 X}(t)=\left\{s_{201}, s_{202}, \cdots, s_{20 n}\right\}, \\
& \boldsymbol{p}_{10 X}\left(\psi_{1}, \psi_{2}, \cdots, \psi_{n}\right)=\left\{\boldsymbol{p}_{201}, \boldsymbol{p}_{202}, \cdots, \boldsymbol{p}_{20 n}\right\}, \\
& T_{10 X}(t)=\left\{t_{201}, t_{202}, \cdots, t_{20 n}\right\}, \\
& L_{10 X}(t)=\left\{l_{201}, l_{202}, \cdots, l_{20 n}\right\}, \\
& x_{10 X}(t)=\left\{x_{201}, x_{202}, \cdots, x_{20 n}\right\}, \\
& G_{U 10 X}(t)=\left\{g_{201}, g_{202}, \cdots, g_{20 n}\right\}, \\
& n=11 .
\end{aligned}
$$

Combining the three constrains of $k g, r w, x q$ to $U_{10 X}$ mentioned above, the solution satisfied $X \cap k g \cap r w \cap$ $x q$ is:

$$
\begin{aligned}
& U_{10 X}=\left\{u_{201}, u_{202}, \cdots, u_{20 n}\right\}, \\
& S_{10 X}(t)=\left\{s_{201}, s_{202}, \cdots, s_{20 n}\right\}, \\
& \boldsymbol{p}_{10 X}\left(\psi_{1}, \psi_{2}, \cdots, \psi_{n}\right)=\left\{\boldsymbol{p}_{201}, \boldsymbol{p}_{202}, \cdots, \boldsymbol{p}_{20 n}\right\}, \\
& T_{10 X}(t)=\left\{t_{201}, t_{202}, \cdots, t_{20 n}\right\}, \\
& L_{10 X}(t)=\left\{l_{201}, l_{202}, \cdots, l_{20 n}\right\}, \\
& x_{10 X}(t)=\left\{x_{201}, x_{202}, \cdots, x_{20 n}\right\}, \\
& G_{U 10 X}(t)=\left\{g_{201}, g_{202}, \cdots, g_{20 n}\right\}, \\
& n=4 .
\end{aligned}
$$

In the same way, we can acquire:

$$
\begin{aligned}
& U_{11 X}=\left\{u_{201}, u_{202}, \cdots, u_{20 n}\right\}, \\
& S_{11 X}(t)=\left\{s_{201}, s_{202}, \cdots, s_{20 n}\right\}, \\
& \boldsymbol{p}_{11 X}\left(\psi_{1}, \psi_{2}, \cdots, \psi_{n}\right)=\left\{\boldsymbol{p}_{201}, \boldsymbol{p}_{202}, \cdots, \boldsymbol{p}_{20 n}\right\}, \\
& T_{11 X}(t)=\left\{t_{201}, t_{202}, \cdots, t_{20 n}\right\}, \\
& L_{11 X}(t)=\left\{l_{201}, l_{202}, \cdots, l_{20 n}\right\}, \\
& x_{11 X}(t)=\left\{x_{201}, x_{202}, \cdots, x_{20 n}\right\}, \\
& G_{U 11 X}(t)=\left\{g_{201}, g_{202}, \cdots, g_{20 n}\right\}, \\
& n=8 .
\end{aligned}
$$

Combining the three constrains of $k g, r w, x q$ to $U_{11 X}$ mentioned above, the solution satisfying $X \cap k g \cap r w \cap$ $x q$ is:

$$
U_{11 X}=\left\{u_{201}, u_{202}, \cdots, u_{20 n}\right\},
$$




$$
\begin{aligned}
& S_{11 X}(t)=\left\{s_{201}, s_{202}, \cdots, s_{20 n}\right\}, \\
& \boldsymbol{p}_{11 X}\left(\psi_{1}, \psi_{2}, \cdots, \psi_{n}\right)=\left\{\boldsymbol{p}_{201}, \boldsymbol{p}_{202}, \cdots, \boldsymbol{p}_{20 n}\right\}, \\
& T_{11 X}(t)=\left\{t_{201}, t_{202}, \cdots, t_{20 n}\right\}, \\
& L_{11 X}(t)=\left\{l_{201}, l_{202}, \cdots, l_{20 n}\right\}, \\
& x_{11 X}(t)=\left\{x_{201}, x_{202}, \cdots, x_{20 n}\right\}, \\
& G_{U 11 X}(t)=\left\{g_{201}, g_{202}, \cdots, g_{20 n}\right\}, \\
& n=4 .
\end{aligned}
$$

\section{Conclusions}

In order for a decision to be correct, we need to know the rule of how errors generate or convert. Each object in real world can be indicated as $(u, x)$. Every element $(u, x)$ is expressed as

$$
\begin{aligned}
& \left(U, S(t), \boldsymbol{p}\left(\psi_{1}, \psi_{2}, \cdots, \psi_{n}\right), T(t), L(t),\right. \\
& \left.\left(x(t)=f\left((u(t), \boldsymbol{p}), G_{U}(t)\right)\right), G_{U}(t)\right)
\end{aligned}
$$

from the standpoint of logic theory. An error matrix can be constructed when the seven parameters mentioned above in $(u, x)$ are used as matrix columns. And three error matrixes $X, A, B$ can create an error matrix equation $X A \supseteq B$. Matrix $A$ is express current status and matrix $B$ is expectant status, $X$ is what we want to achieve. $X$ allows us to find a method, in which bad status " $A$ " changes into good status " $B$ ".

Based on the error matrix equation, which is a kind of mathematical tool to be used to describe error itself and the transformation rules of errors, this paper proposes a new kind of error matrix equation named "containingtype error matrix equation". The method of solving, existence and form of solution for this type of equation have been presented in this paper. Our research provides a new method of decision making.

\section{REFERENCES}

[1] K. Z. Guo and S. Y. Liu, "Introduction for the Theory of Error-Eliminating," Advances in Modelling \& Analysis A: Mathematical, General Mathematical Modelling, Vol. 39, No. 4, 2002, pp. 39-66.

[2] K. Z. Guo and S. Q. Zhang, "Theory of Error Sets," Central South University Press, Changsha, 2001.

[3] K. Z. Guo, "Error Logic," Science Press, Beijing, 2008.

[4] K. Z. Guo and S. Y. Liu, "Fuzzy Error System with Change of Time and Space-The Effect of Change of Time and Space on Decision Making," Advances in Modeling and Analysis B, Vol. 45, No. 3, 2002, pp. 49-60.

[5] K. Z. Guo and S. Y. Liu, "Logical Relationship between Fuzzy Error Logical Decomposition Words and a Pre-Decomposed No Words," Advances in Modelling \& Analysis A: Mathematical, General Mathematical Modelling, Vol. 39, No. 4, 2002, pp. 1-15.

[6] K. Z. Guo and S. Y. Liu, "Exploration and Application of Redundancy System in decision Making Relation between Fuzzy Error Logic Increase Transformation Word and Connotative Model Implication Word," Advances in Modelling \& Analysis A: Mathematical, General Mathematical Modelling, Vol. 39, No.4, 2002, pp. 17-27.

[7] M. Li and K. Z. Guo, "Research on Decomposition of Fuzzy Error Set," Advances in Modelling \& Analysis A: Mathematical, General Mathematical Modelling, Vol. 43, No.2, 2006, pp. 15-26.

[8] X. L. Min, K. Z. Guo, "Knowledge Model Based on Error Logic for Intelligent System," World Congress on Computer Science and Information Engineering, Los Angeles, 31 March-2 April 2009, pp. 216-220.

[9] X. L. Min and K. Z. Guo, "A Knowledge Discovery Method Based on Error Matrix Equation," The Sixth International Conference on Fuzzy Systems and Knowledge Discovery, Tianjin, 14-16 August 2009 pp. 151-155. doi:10.1109/FSKD.2009.104

[10] X. L. Min and K. Z. Guo, "A Primary Probe on the Application of Error-Eliminating Theory in Urban Traffic Jam Management System," Advances in Systems Science and Applications, Vol. 9, No. 3, 2009, pp. 486-493. 